\title{
Pre-Crash Braking System-PCBS
}

\author{
Hawar Bahzad Ahmad ${ }^{1}$, Zakiya Ali Nayef ${ }^{2}$ \\ ${ }^{1,2}$ Department of Computer Science, Nawroz University, Duhok, Kurdistan Region - Iraq
}

\begin{abstract}
In computer vision the detection of Pedestrian assumed as a key problem, by various applications that have the possibility to impact positively life quality. In recent years, Growing the number of methods of pedestrians detection in monosyllabic images. This paper presents a system that accurately detects pedestrians and provides the driver with informative warnings by using the Pre-Crash Braking System-(PCBS), which is use sensor and the brake-by-wire actuator. One of the main goals of transportation safety we presented in this paper to protect pedestrians from crashes with vehicles and reduce their risk on roadways. We chose to focus on detection by the driver and assume perfect detection by the system according to that we used (use-case) diagram to shows the different interactions available to users of the PCB System. This proposed system minimizes accident possibilities and reduction of accident impact on pedestrians which keeps the driver alert to dangerous situations even in moments of driver distraction due to the functionalities of the system modules.
\end{abstract}

Keywords: Pre-Crash, PCBS, Tracking.

\section{Introduction}

Pedestrian safety is a global concern. A World Health Organization report [7] describes traffic accidents as one of the major causes of death and injuries around the world, accounting for an estimated 1.2 million fatalities and 50 million injuries. In low-income countries, a large majority of deaths are not the vehicle occupants but the vulnerable road users (VRUs), consisting of pedestrians, bicyclists, two wheelers, and other small vehicles. In high-income countries, pedestrian fatalities are relatively lower but still represent large societal and economic costs to the nations. Although there has been a decline in the population of walking pedestrians but it still remain a very common employed mode of traveling. People of almost all ages, both sexes and all walks of life to protect pedestrians from crashes with motor vehicles and reduce their risk on roadways is one of the main goals of transportation safety. Learning about and studying pedestrian crashes, especially those related to the deaths of pedestrians, is a part of the effort to reach that goal. This project describes pedestrian crash

Academic Journal of Nawroz University

(AJNU) Volume 7, No 4 (2018).

Regular research paper : Published 21 December 2018

Corresponding author's e-mail : hawar.duhoki@yahoo.com

Copyright @2017 Hawar Bahzad Ahmad1, Zakiya Ali Nayef ${ }^{2}$. This is an open access article distributed under the Creative Commons Attribution License. fatalities and injuries in the United States by providing statistics and crash characteristics. The statistics cover pedestrian crash deaths and injuries nationwide from 1997 to 2006. Results of the data analysis are reported. Figure 1 below shows the distribution of pedestrian fatalities by crash type and calendar year from 1997 to 2006. The proportion of pedestrian deaths in singlevehicle (SV) crashes did not show any significant change between 1997 and 2006. Pedestrians are often killed in SV crashes. An overwhelming majority of pedestrian deaths - more than 90 percent - are in SV crashes.

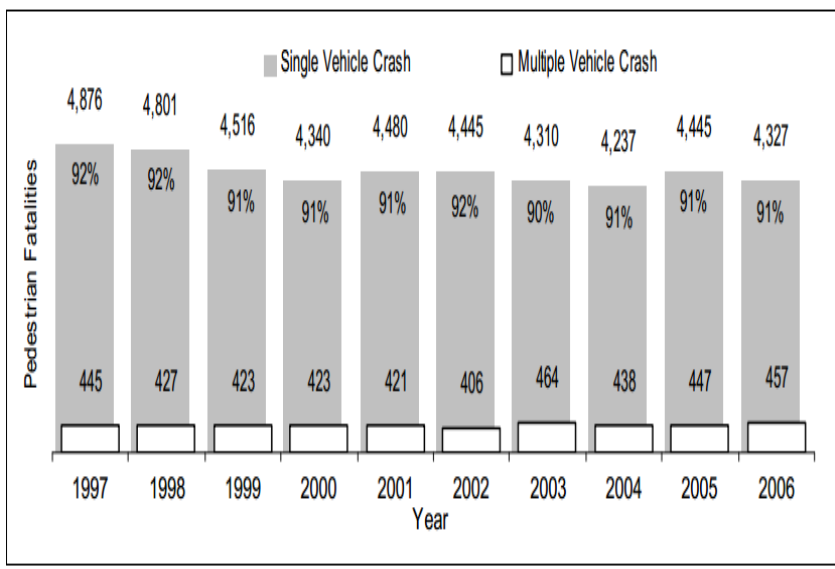

Fig (1) : Pedestrians Killed by Crash Type and Year

Source: FARS 1997-2005 (Final), 2006 (ARF)

Figure 2 below shows that the pedestrian crash probability has not significantly changed over the past decade, yet the pedestrian fatality probability 
increased more than one third - from 5.3 percent in 1999 to 7.1 percent in 2006. It has steadily increased since 1999.

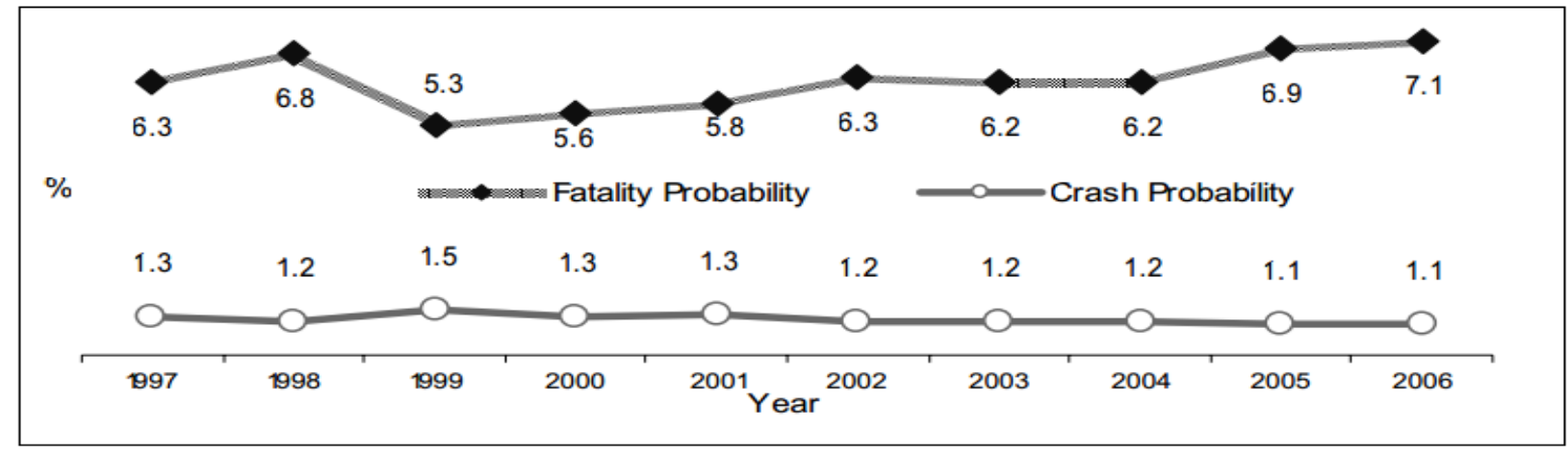

Fig (2) : Pedestrian Fatality Probability and Crash Probability by Year Source: FARS 1997-2005 (Final), 2006 (ARF) and GES 1997-2006

Pedestrians over age 64 accounted for more than 21 percent of the pedestrians killed, and children under the age of 16 accounted for about 10 percent of the fatalities. The $70+$ age group had the highest pedestrian fatalities among all individual age groups (17 percent), and the 40-to-44 and 45-to-49 age groups had the second highest pedestrian fatalities ( 9 percent). The percentages by age group of pedestrians killed are shown in Figure 3.

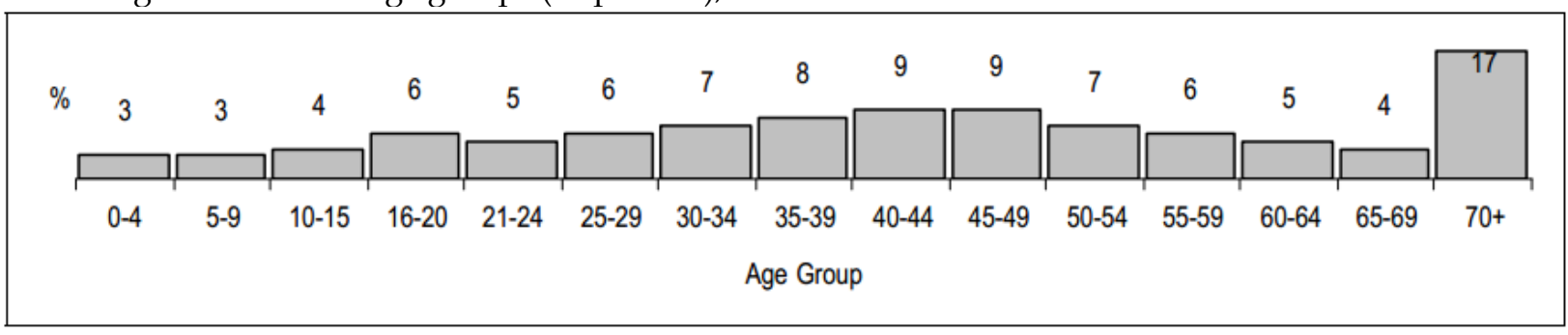

Fig (3) : Distribution of Pedestrian Fatalities by Age Group Source: FARS 1997-2005 (Final), 2006 (ARF)

\subsection{Problem Statement}

Basing on previous studies conducted on the fatality risk estimation on pedestrians knocked down by cars, it can be deduced that the speed at which a car is travelling determines the accident fatality level. The table below shows previous works on different vehicle speeds and estimation on the fatality risk estimation in previous publications on fatality risk for pedestrians struck by passenger cars.

\begin{tabular}{|c|c|c|c|c|}
\hline & Years of data & $30 \mathrm{~km} / \mathrm{h}$ & $50 \mathrm{~km} / \mathrm{h}$ & $70 \mathrm{~km} / \mathrm{h}$ \\
\hline $\begin{array}{l}\text { Anderson et al. } \\
\text { (1997) }\end{array}$ & 1978 & $8 \%$ & $85 \%$ & $100 \%$ \\
\hline Ashton (1982) & $1965-1979$ & $\approx 5 \%$ & $\approx 45 \%$ & $\approx 95 \%$ \\
\hline Pasanen (1992) & 1965-1979 & $6 \%$ & $40 \%$ & $94 \%$ \\
\hline Yaksich (1964) & $1958-1963$ & $\approx 22 \%$ & $\approx 65 \%$ & $100 \%$ \\
\hline Cuerden et al. (2007) & $2000-2007$ & $\approx 2 \%$ & $\approx 12 \%$ & $\approx 33 \% *$ \\
\hline Davis $(2001)^{* \star}$ & 1965-1979 & $1 \%$ & $7 \%$ & $51 \%$ \\
\hline $\begin{array}{l}\text { Hannawald \& Kauer } \\
(2004)\end{array}$ & $1991-2003$ & $4 \%$ & $14 \%$ & $39 \%$ \\
\hline Oh et al. $(2008 b)^{\star \star \star *}$ & 2003-2005 & $7 \%$ & $34 \%$ & $77 \%$ \\
\hline
\end{tabular}

Fig (4) : Fatality risk estimation [11]

*Estimate was based on private communication with Cuerden and was not given in Cuerden ed al. (2007).

**Risk estimates regard pedestrians in the ages 15 to 59 years.

***Striking vehicles included passenger cars, SUV's, vans, trucks and busses. 
The study is focused on an age group between 15 to 59 years in different countries because the authors believed that under normal circumstances, the ideal age for one to cross the road without assistance is between 15 and 59 years of age. Below the stated age group was considered a minor and above the group was considered an elderly person who might need assistance.

From the table above, it can be concluded that the fatality risk of an accident is less when the speed of the vehicle is lower.

\subsection{Objective}

The objective of the Pre-Crash Braking System-PCBS is to accurately detect pedestrians and provide the driver with informative warnings. In the eyes of the driver, the end product of a good system provides a timely warning and, possibly, additional information such as the distance of the pedestrian from the car. Although generic image processing algorithms have been addressing similar goals for many years, there are several problems that are unique to image processing in automotive applications.

In the present experiment, we chose to focus on detection by the driver and assume perfect detection by the system

In light of the above, this experiment addressed one main issue:

- Minimizing accident possibilities and reduction of accident impact on pedestrians

\section{PROPOSED SYSTEM AND FEATURES IN GENERAL}

\subsection{System Prototype / Perspective}

Pre-Crash Braking System (PCBS) is a system that gives autonomous braking ability to vehicles by using the pedestrian sensor and the brake-by-wire actuator. It recognizes and responds to possible pedestrian collisions by electronically controlling the brakes to decrease speed.

The PCBS countermeasure system is intended to provide drivers with the latest information available for improving the safety and mobility of pedestrian. This pedestrian safety system warns drivers of potential collision, it can help save lives by warning drivers of potential collisions and decreasing vehicle speed.

An active pedestrian safety system that warns a driver of a potential collision, and automatically decreases the speed of the vehicle to mitigate (or even avoid) the collision, has significant potential to help improve survival rates

PCBS is developed in such a way that the vehicle's sensor radar is capable of detecting and tracking pedestrians at distances of over $50 \mathrm{~m}$, even in challenging urban scenarios. If a pedestrian is detected, sophisticated risk assessment algorithms are employed to determine the probability of a collision by taking into account the predicted paths of the vehicle and the pedestrian. Then a warning is issued, or a braking manoeuvre is initiated, with the aim of slowing the vehicle and reducing the severity of the impact. To reduce false collision warnings and false collision mitigation braking events, a second confirmation of a critical collision trajectory with the pedestrian is required by radar.

The user interface has the following constraints:

- Buttons to activate and deactivate the system.

The hardware systems have the following constraints: The pedestrian sensor and the vehicle's current and initial velocities are the only sources of information for the controller, aside from the enable/disable buttons which can activate/deactivate it. No other inputs may be accepted. These inputs are channelled over a physical wire, so a malicious "soft" hack of the PCB system is not possible, only a hardware hack could occur.

- The pedestrian sensor sends a packet every $100 \mathrm{~ms}$ of all pedestrians in its view.

- The packet contains speed, direction, and location relative to the car.

- Once the vehicle is turned on, the PCB system is always running unless it is deactivated by the driver or an error occurs. On an error, the PCB system must deactivate and notify the driver. Additionally, the autonomous driving system as a whole cannot operate without the PCB system, so must also deactivate.

- An actual sensor has a limitation on the number of pedestrians it can detect at once. It is assumed to be fairly large; that is, it is quite unlikely for more pedestrians to be in view than this maximum

The software interface has the following constraints:

- Every pedestrian has a state of alert (near, far, etc.).

- The controller's overall state becomes the state of the "worst" pedestrian.

- "Worst" pedestrian is the pedestrian in the most imminent danger.

- The PCB system must ignore erroneous packets from the pedestrian sensor.

- The PCB system should never put the driver or the occupants of the vehicle at risk.

Additional constraints:

The system must have sufficient memory to store the maximum number of pedestrians that the sensor can detect as well as sufficient working space. On the whole, this will be fairly small amount, especially compared to what modern computing systems have. 


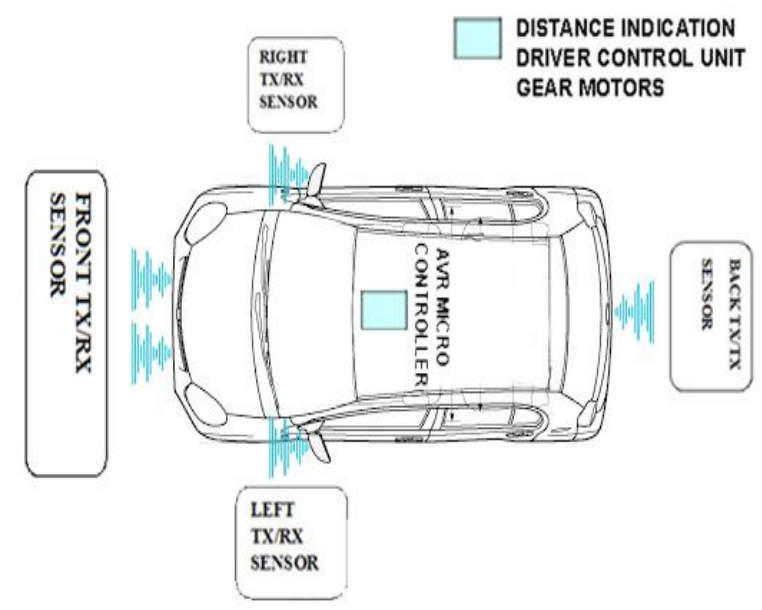

Fig (5) : PCBS Sensor Location in the car

2.2 System Features and Functions

This section of this SRS specifies all major functions of the PCB system with respect to the customer-supplied specifications such as (System On, System Off,
Detection, Obstacle Near, Obstacle Far, Normal, Hard Stop, Warning and Manual Override).

\section{SYSTEM REQUIREMENTS}

The system requirement consists of two parts which are the hardware requirement and software requirement. Contains Hardware Requirement (Avr Microcontroller Processor, Power Supply Unit, Indication Display, Gp2d12 Distance Sensor and Buzzer). And Software Requirements (Avr studio4, Java Program and Android Eclipse Emulator). Also Performance Requirement (Accuracy, Audio feedback and Visual feedback).

\section{SYSTEM MODULE}

The figure below shows the five modules of the system, how various warning and assessment is carried out in the system. It shows the reaction time for a given distance decreases with vehicle speed such as (Sensor Tracking, Feedback, Driver State, Automatic Braking and System Restart).

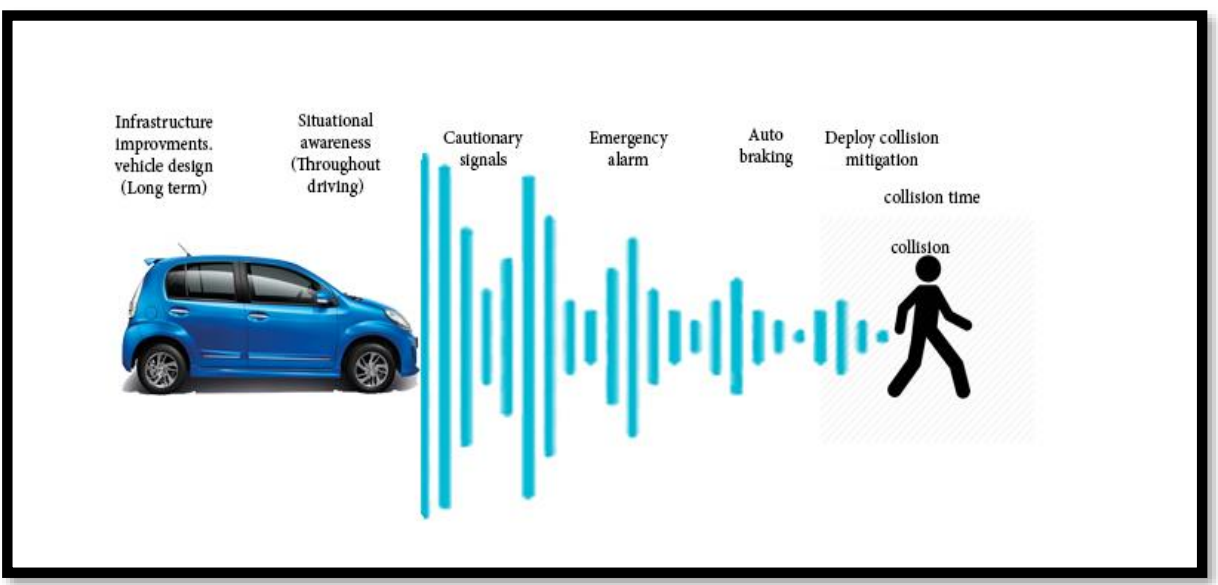

\section{PARAMETER OF THE CAR}

Fig (6) : Timeline of pedestrian protection measures
Perodua Myvi specifications in the study. The diagram below shows the Perodua Myvi measurements from the front, back and side view.

project. Below is a table showing some of the relevant
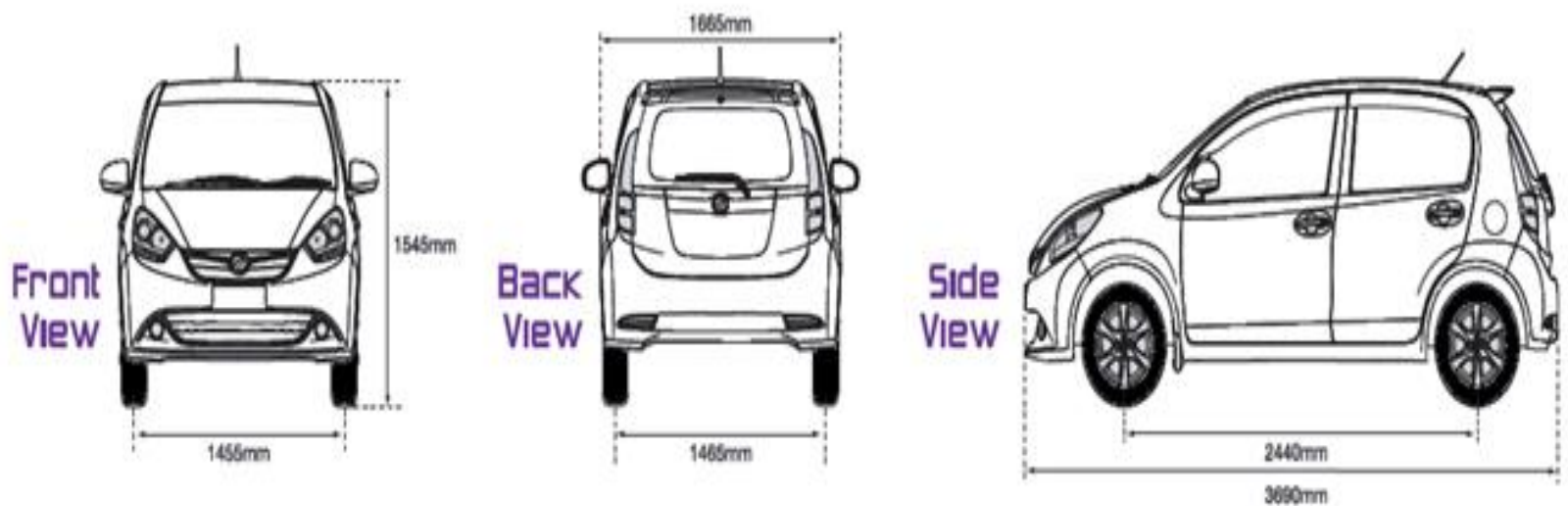

Fig (7) : Perodua Myvi test base car [9] 
Table (1) : Myvi Car Specification

\begin{tabular}{|l|l|}
\hline \multicolumn{2}{|l|}{ Dimension and Weight } \\
\hline Length $(\mathrm{mm})$ & 3690 \\
Width $(\mathrm{mm})$ & 1665 \\
Height $(\mathrm{mm})$ & 1545 \\
Wheelbase $(\mathrm{mm})$ & 2440 \\
Front seat distance to ground $(\mathrm{mm})$ & 595 \\
Minimum road clearance $(\mathrm{mm})$ & 160 \\
Seating capacity & 5 \\
& \\
\hline Engine & \\
\hline Valve Mechanism & DOHC 16V with DVVT \\
Total displacement (cc) & 1298 \\
Max torque (Nm/rpm) & $117 / 4400$ \\
\end{tabular}

In the development of the system prototype, two scenarios were created to enable visualization on how the system will work. The two scenarios include; the car travelling at a speed of $50 \mathrm{~km} / \mathrm{hr}$ and the same vehicle travelling at $150 \mathrm{~km} / \mathrm{hr}$. The distance between the vehicle and the obstacle remained constant.

Assumptions made

- The vehicle tires were at least $90 \%$ new at the time

- The road was flat and dry asphalt

- There was only one person in the car

The system sensors can detect and object that is at a distance of 50meters, at this point the driver is alerted about the obstacle ahead. If the driver does not take action within the 30meter allowance, the system automatically brakes for the driver. For a typical vehicle with a displacement of 1300 cubic centimetres like the above mentioned, it takes the vehicle about 70 meters to decelerate from $100 \mathrm{~km} / \mathrm{hr}(100,000 \mathrm{~m} / \mathrm{hr})$ to a complete stop when sudden brakes are triggered. Therefore the deceleration rate is $0.0005623 \mathrm{~km} / \mathrm{hr}$ $(0.5623 \mathrm{~m} / \mathrm{hr})$.

\subsection{Scenario 1}

In this scenario, the vehicle is moving at a speed of $50 \mathrm{~km} / \mathrm{hr}$ and senses an obstacle 50meters ahead, the automatic braking will only start when the car is $20 \mathrm{~m}$ from contact. The vehicle in this scenario will be able to come to a complete halt in 14 meters. This leaves a distance of $5.5 \mathrm{~m}$ from the vehicle to the obstacle.

This is achieved by:

$$
\begin{aligned}
& v^{2}=u^{2}+2 a s \\
& \mathrm{v} \text { = final velocity (it must be } 0 \text { to imply a stop) } \\
& \mathrm{u} \quad=\quad \text { initial velocity } \\
& \mathrm{s}=\text { displacement of the vehicle } \\
& \mathrm{a}=\text { acceleration (when negative it implies deceleration) }
\end{aligned}
$$

Working out the rate of deceleration; if $100 \mathrm{~km} / \mathrm{hr}$ stops in $56.23 \mathrm{~m}$, therefore the rate at which the car was decelerating is:

$$
\begin{gathered}
\mathrm{v}^{2}=\mathrm{u}^{2}+2 \mathrm{as} \\
0=100^{2}+2(\mathrm{a} * 0.0563) \\
0=10,000+0.1126 \mathrm{a} \\
-10,000=0.1126 \mathrm{a} \\
\mathrm{a}=-\mathbf{8 8}, \mathbf{8 0 9 . 9} \mathbf{~ k m} / \mathbf{h} \mathbf{r}^{2}
\end{gathered}
$$

The deceleration has to be less than zero because it is a reduction in speed. Therefore for a vehicle travelling at $50 \mathrm{~km} / \mathrm{hr}$ :

$$
\begin{gathered}
\mathrm{v}^{2}=\mathrm{u}^{2}+2 \mathrm{as} \\
0=50^{2}+2(-88,809.9 * \mathrm{~s}) \\
0=2500-177,619.8 \mathrm{~s} \\
177,619.8 \mathrm{~s}=2500 \\
\mathbf{s}=\mathbf{0 . 0 1 4} \mathbf{~ k m}(\mathbf{1 4} \mathbf{~ m})
\end{gathered}
$$

$14 \mathrm{~m}$ is less than $20 \mathrm{~m}$ at which the brakes were triggered meaning the car will come to a complete halt before reaching the obstacle.

\subsection{Scenario 2}

If the driver was at a speed of $150 \mathrm{~km} / \mathrm{hr}$ and basing on the same rate of deceleration as the one above, the car will actually hit the obstacle. This can be proven using the same formula.

$$
\begin{gathered}
\mathrm{v}^{2}=\mathrm{u}^{2}+2 \mathrm{as} \\
0=150^{2}+2(-88,809.9 * \mathrm{~s}) \\
0=22,500-177,619 \mathrm{~s} \\
177.619 \mathrm{~s}=22,500 \\
\mathbf{s}=\mathbf{0 . 1 2 6} \mathbf{~ k m}(\mathbf{1 2 6} \mathbf{~ m})
\end{gathered}
$$

$126 \mathrm{~m}$ is greater than $20 \mathrm{~m}$ at which the brakes were triggered thus the car will come to a complete halt after hitting the obstacle. To find out the speed at which the car was moving at the point of impact the same formula can be used. However the displacement is reduced by $20 \mathrm{~m}$. 


$$
\begin{gathered}
\mathrm{v}^{2}=\mathrm{u}^{2}+2 \text { as } \\
\mathrm{v}^{2}=150^{2}+2(-88,809.9 * 0.106) \\
\mathrm{v}^{2}=22,500-18,827.7 \\
\mathrm{v}^{2}=3672.3 \\
\mathbf{v}=\mathbf{6 0 . 6} \mathbf{~ k m} / \mathbf{h r}
\end{gathered}
$$

It can be noticed that use of the system reduced the speed of the vehicle by more than half therefore also reducing the fatality rate of impact on the pedestrian.

\subsection{Modeling Requirement}

Included are models describing a possible software system to meet the requirements of the PCBSystem. Included are a use-case diagram, a class diagram, and a object state diagram. In general, lowercase terms (e.g. "pedestrian" and "vehicle") refer to the actual, physical objects, whereas uppercase terms (e.g. "Pedestrian" and "Vehicle") refer to the actual object's software representation.

\subsection{Modelling Requirement Description}

Based on interaction with the customer, the list below is a summation of modelling requirements description. 1. The system will consist of a pedestrian sensor, a controller, and a brake-by-wire actuator.

2. The system must attempt to avoid collisions when such avoidance is possible. Detected pedestrians and obstacles will be organized into two categories: a) Obstacle near, and b) Obstacle far. If no pedestrians are detected, the system takes the Normal state.

3. After each packet received from the sensor, the system must continuously recalculate the obstacle's distance and re-categorize the pedestrian based on that information, and respond appropriately.

4. The system must return to steady-state velocity after a braking. This may only occur when the pedestrian is categorized as "Normal" system state.

5. The system should not make hard stops when a collision is unlikely or impossible (obstacle far).
6. The driver should be able to disable the system in times of malfunctioning or in cases of system failure. This will disable the system.

7. In the "obstacle near" state $(<20 \mathrm{~m})$, hard stops become a possibility. Because this is irritating to the driver and occupants of the vehicle, this should only occur when necessary. Additionally, a warning signal from the infrared distance measuring sensor must not cause a hard stop.

8. A request to the brake-by-wire actuator which duplicates the currently-taken action (e.g., if the driver is already braking) the request should be ignored by the micro controller. The brake-by-wire actuator should NOT release, and then reapply the brakes.

9. Any input provided by the driver must override any actions taken by the system. Additionally, a manual ignore button will be provided, which also shuts down the entire autonomous driving system.

\subsection{Class Diagram}

The following class diagram shows the structure of the PCBS system by displaying its classes. The classes have different operations and member variables which may or may not be accessible to other classes. Associations between different classes are displayed via a solid line, with text labels giving the role (how they relate) and multiplicity (how many objects of the class may be involved in the relation) of each particular class in the association. Aggregation is shown much like association is shown, but with a diamond touching one class to display that this class is made up of items from a "smaller" class that the line connects to. This particular diagram also contains an enumeration, which is a list of values that a variable can take. 


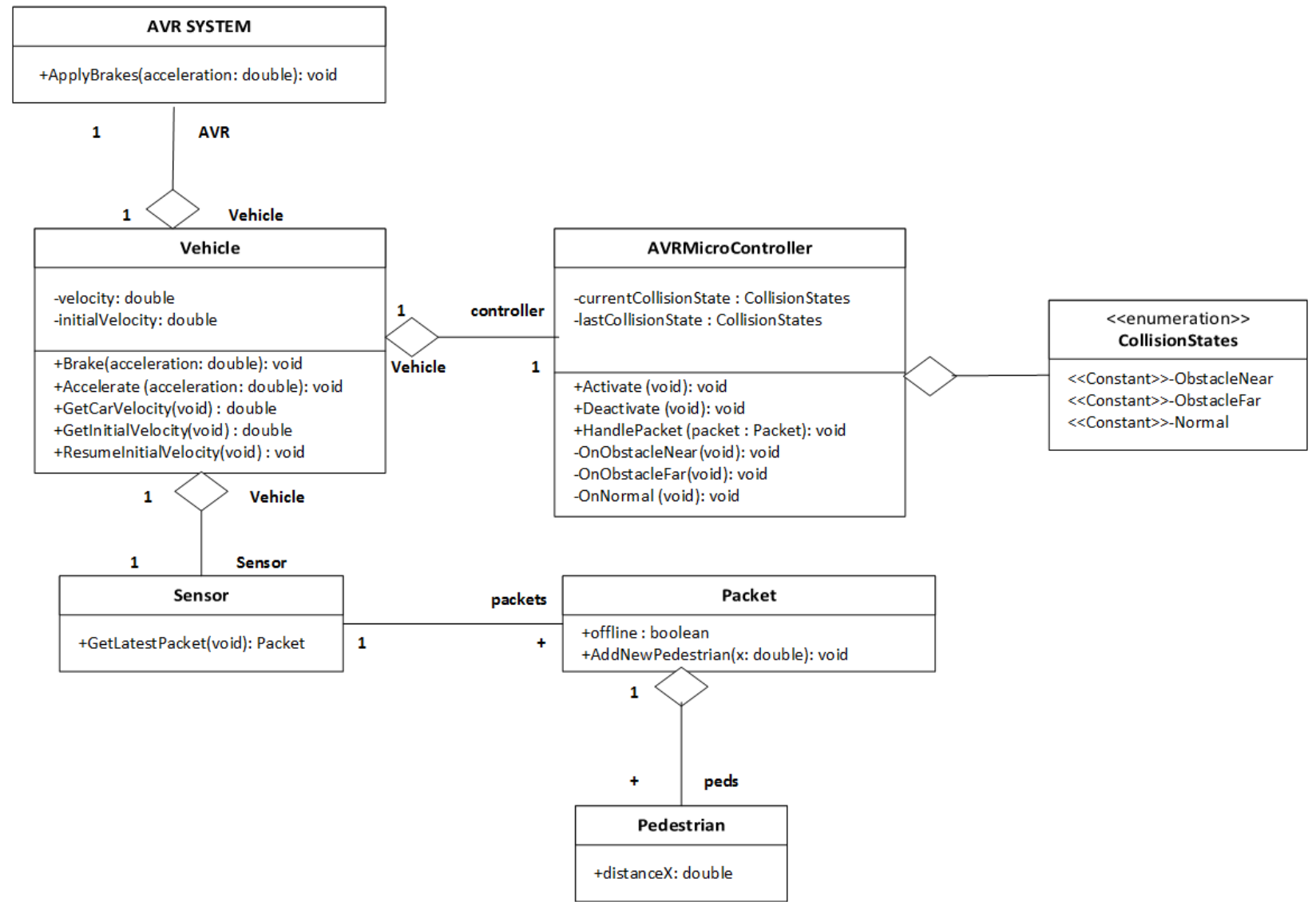

Fig (8) : Class Diagram

In this class diagram (Figure 8), the Vehicle owns all the other objects either directly or indirectly, and also would contain the main thread of execution. Packets containing Pedestrian data are created by the Sensor, collected by the Vehicle and then given to the AVRMicroController, which decides what actions to take based on the Pedestrian data, and instructs the Vehicle to execute those actions. The Vehicle forwards braking requests to the AVR SYSTEM (brake-by-wire actuator), and is itself responsible for acceleration requests. Below are several tables (called a "data dictionary") describing each class and its attributes, operations, and relations in detail.

\subsection{Use Case Diagram}

The following use-case (Figure 9) diagram shows the different interactions available to users of the PCB System. The stick figure of a person symbolizes a user or other external actor, and the arrows protruding from them reach the ovals which label the high-level different actions that a user can take to affect the system's behaviour.

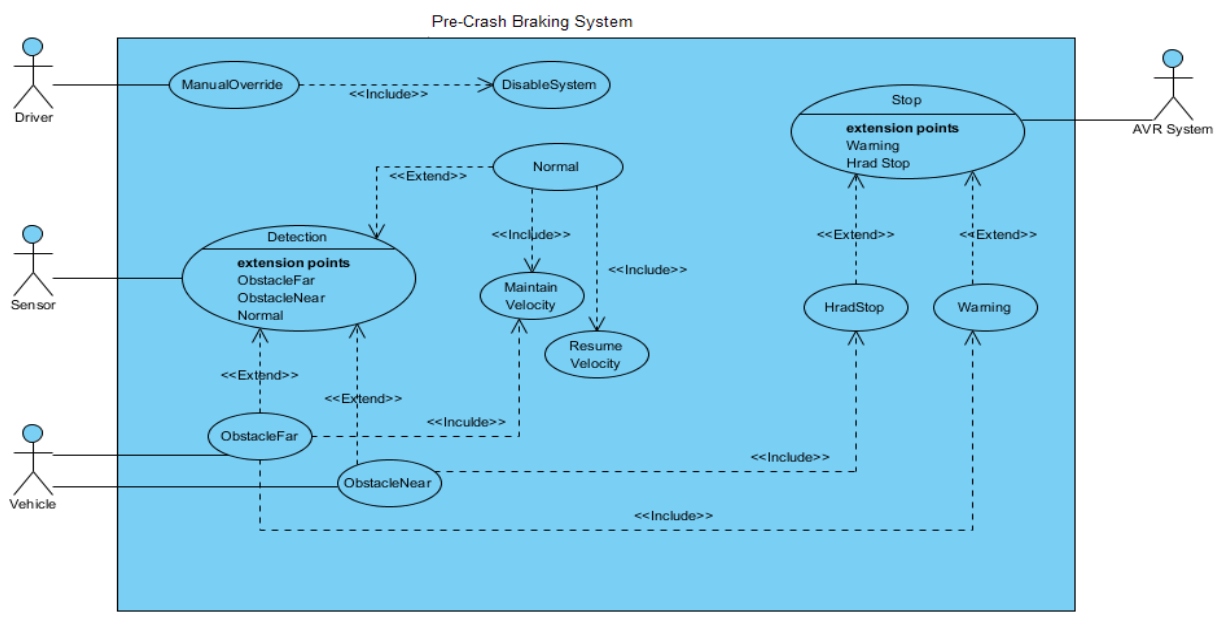

Fig (9) : Use Case Diagram 
This use case diagram includes 14 main use cases, described in detail in the tables below in cross reference to (Modelling Requirement Description in 6.1)

\subsection{State Machine Diagram}

The following state diagram (Figure 10) shows the different states that the PCB System switches to base on events. These events are decided upon based on the data provided by the Sensor. Any rectangle with a name inside depicts a single state, and arrows between states are labelled with the events that would occur to shift the system from one state to another. Events may contain actions which are executed. In this particular diagram, the states all have entry actions, which are always executed upon entering the state.

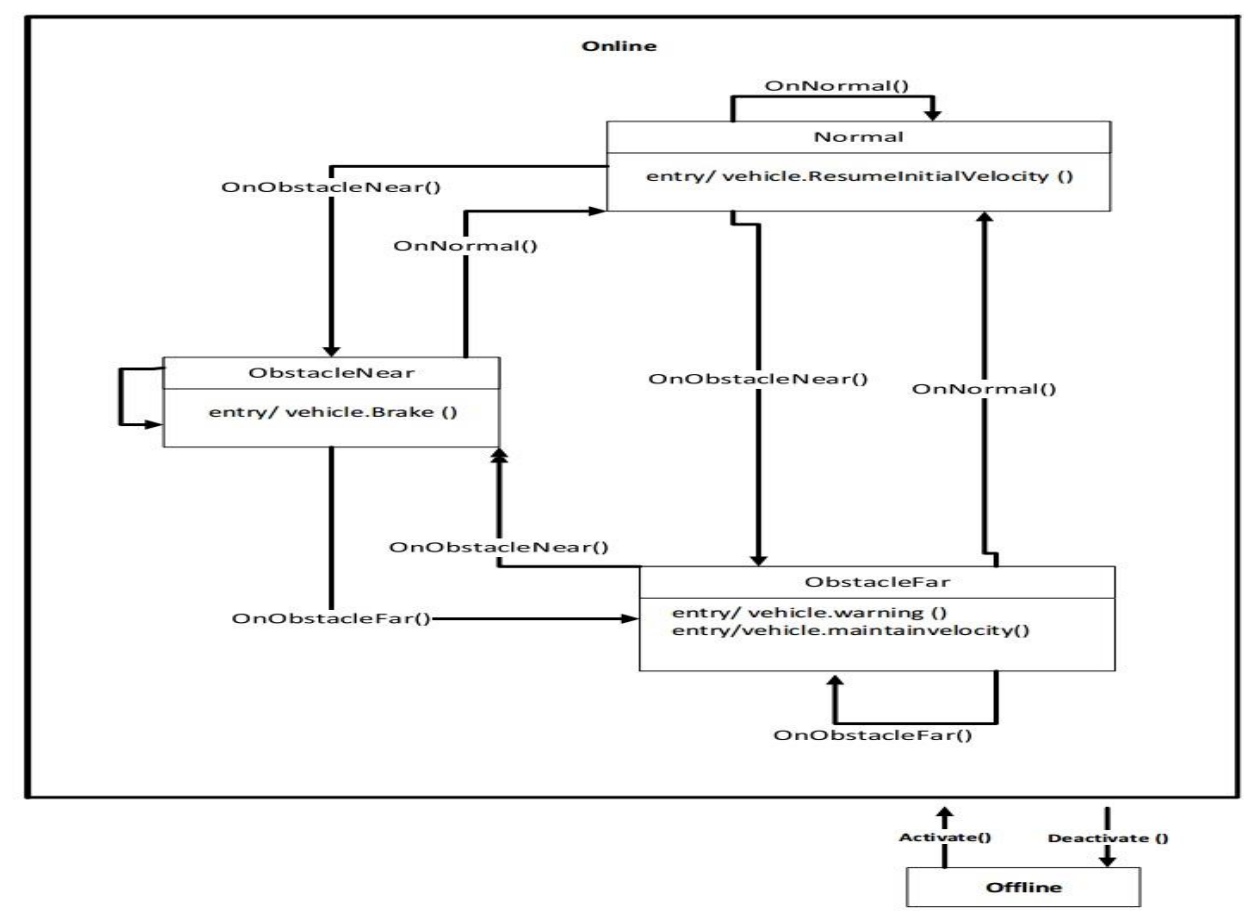

Fig (10) : State Machine Diagram

At first glance the state diagram appears rather complicated but is actually fairly simple. Each of the four main system states can transition to the other three or itself. On each transition, an entry action occurs. Upon entering ObstacleNear or ObstacleFar, a braking amount is calculated to apply. Upon entering Normal , the Vehicle is instructed to resume its initial velocity. If it is already at its initial velocity, the Vehicle will simply take no action.

\subsection{SYSTEM PROTOTYPE}

A prototype of the PCBSystem is created in order to demonstrate a possible decision engine for the AVRMicroController. Given the standard scenarios in section 5.1 and 5.2 , the prototype will simulate what would occur in such a scenario at each time when the sensor senses the pedestrian. 


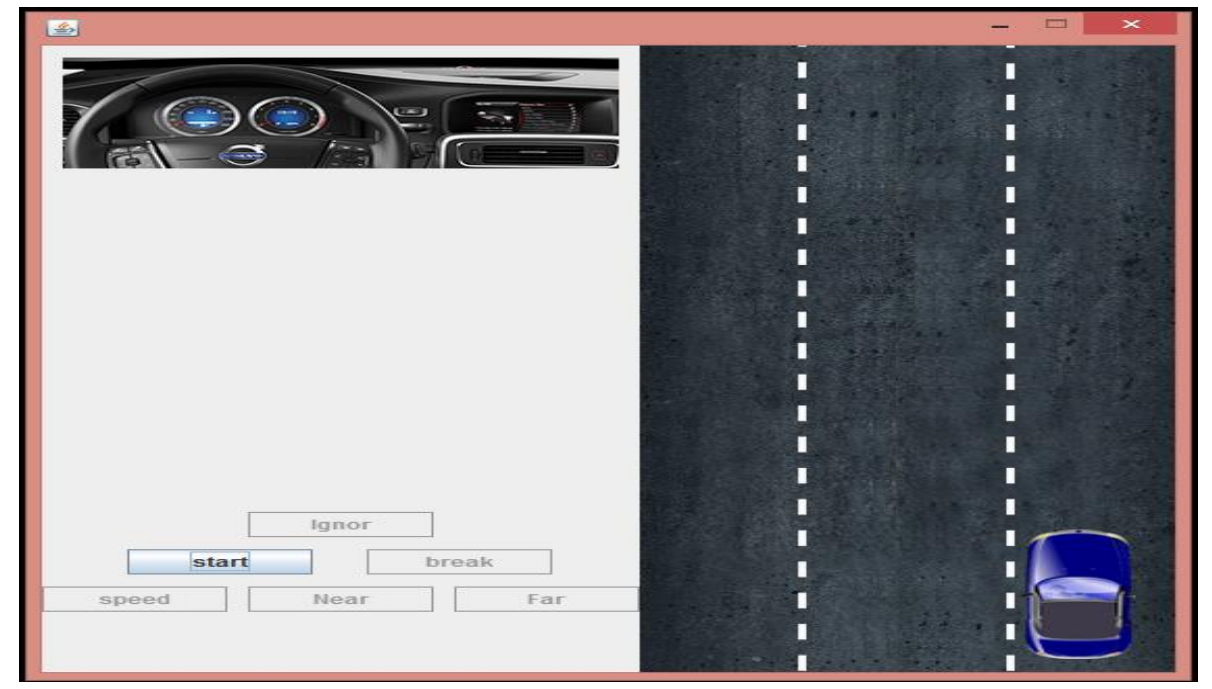

Fig (11) : Initial State of PCBS

Start button, Warning display dashboard, Ignore button, Speed button, Near button, Far button and Break button.

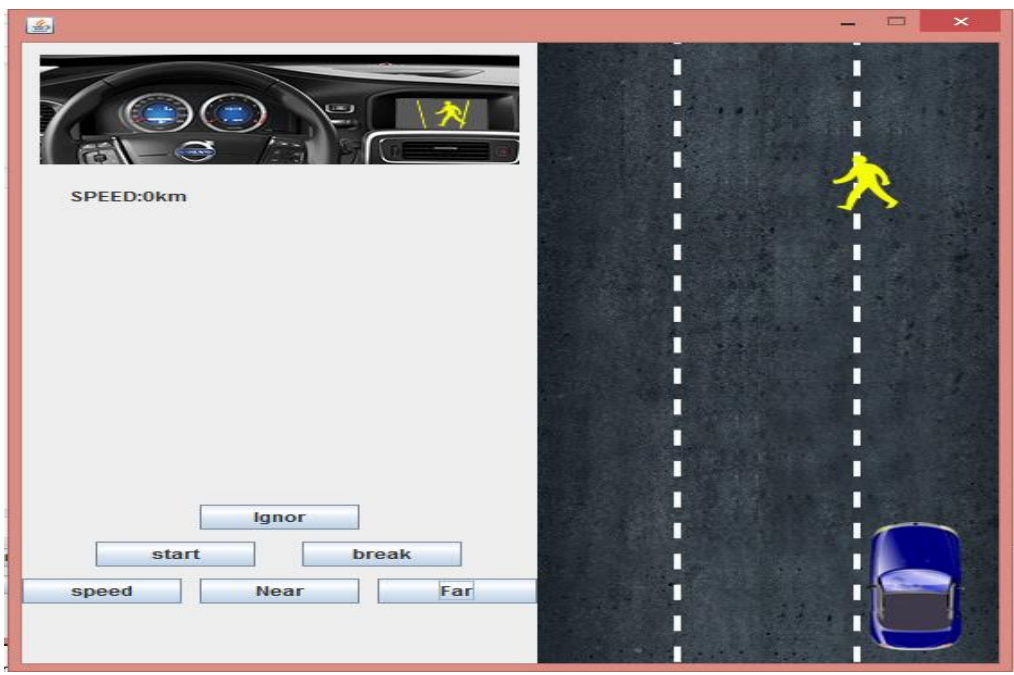

Fig (12) : Dashboard displaying warning to the driver after detecting a far pedestrian (Section 4.0)

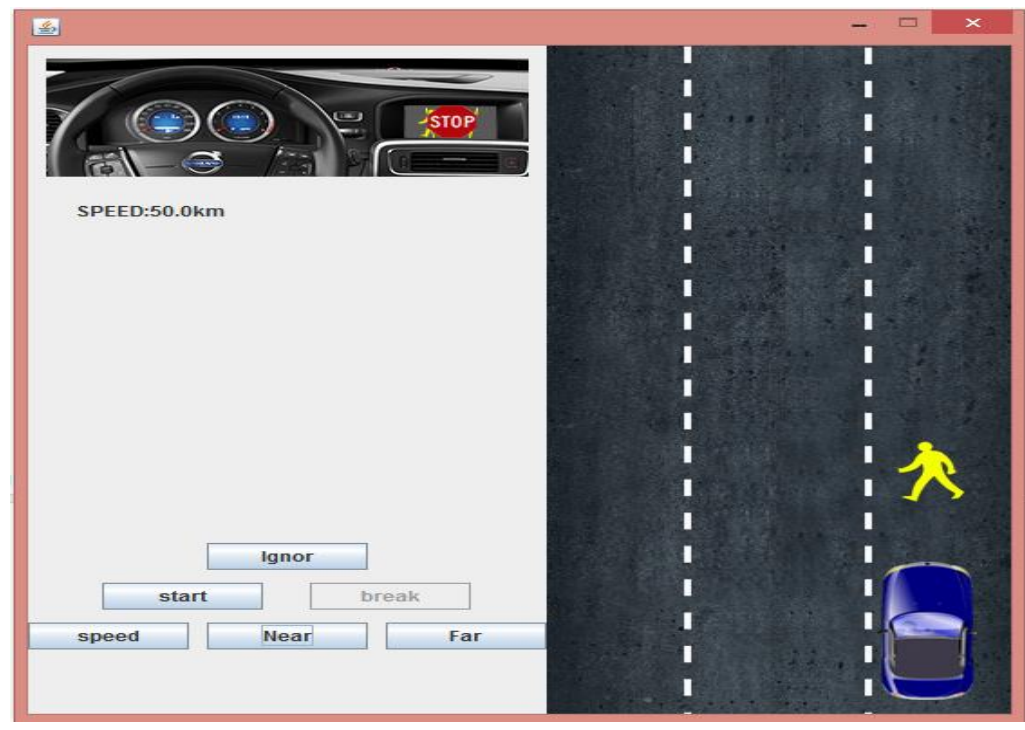

Fig (13) : Dashboard displaying warning to the driver after detecting a near pedestrian (Section 4.0) 


\subsection{CONCLUSION}

There is no simple universal solution that would reduce pedestrian casualties because of the large numbers of pedestrians and high traffic flows. Pedestrians are the most vulnerable road users, and therefore, they require maximum protection on the road. The large number of fatalities and injuries show the importance of developing pedestrian protection systems. This proposed system minimizes accident possibilities and reduction of accident impact on pedestrians which keeps the driver alert to dangerous situations even in moments of driver distraction due to the functionalities of the system modules. The design of this system is composed of loosely integrated modules that react to different ways the vehicle may move, and will be able to accommodate future inclusion of new modules without problem.

\section{REFERENCES.}

[1] Pedestrian Protection Systems: Issues,Survey, and Challenges. IEEE TRANSACTIONS ON INTELLIGENT TRANSPORTATION SYSTEMS, VOL. 8, NO. 3, SEPTEMBER 2007413

[2] Y. Abramson and B. Steux, "Hardware-friendly pedestrian detection and impact prediction," in Proc. IEEE Intell. Veh. Symp., Jun. 2004, pp. 590-595.

[3] G. Antonini, S. V. Martinez, M. Bierlaire, and J. P. Thiran, "Behavioral priors for detection and tracking of pedestrians in video sequences," Int.J. Comput. Vis., vol. 69, no. 2, pp. 159-180, 2006.

[4] M. M. Meinecke, M. A. Obojski, M. Töns, and M. Dehesa,"SAVE-U: First experiences with a pre-crash system for enhancing pedestrian safety," in Proc. 5th Eur. Congr. Intell. Transp., Jun. 2005.

[6] http://www.motortrader.com.my/news/top-50models-in-may-2012/

[7] M. Peden, R. Scurfield, D. Sleet, D. Mohan, A. A. Hyder, E. Jarawan, and C. Mathers, Eds. , World Report on Road Traffic Injury Prevention.Geneva, Switzerland: World Health Organization, 2004.

[8] NHTSA's National Center for Statistics and Analysis, 1200 New Jersey Avenue SE., Washington, DC 20590 "http://www-nrd.nhtsa.dot.gov/Pubs/810968.PDF"

[9] Top 50 models in May 2012|Motor Trader Car News. Bibliography: Motortrader.com.my. 2012. Top 50 models in May 2012|Motor Trader Car News “http://www.motortrader.com.my/news/top-50models-in-may-2012 “

[10] Forensicdynamics.com. 2013. Vehicle Stopping Distance Calculator.http://forensicdynamics.com/stoppingdistance-calculator

[11] Erik, R. Ulrich S. “Accident analysis \&prevention: Pedestrian fatality risk as a function of car impact speed," Science Direct [online] 41 (3) 2009. http://www.ittc.ku.edu/ krsna/citing.htm

[12] Functional Algorithm for Automated Pedestrian Collision Avoidance System." Continental Automotive
Systems, Advanced Engineering, 12 Oct. 2012. http://www.cse.msu.edu/ cse435/Projects/F2012/De scriptions/APCA.pdf

[13] Llorca, David F., Vicente Milanes, Ignacio P. Alonso, Miguel Gavilan, Ivan G. Daza, Joshue Perez, and Miguel A. Sotelo. "Autonomous Pedestrian Collision Avoidance Using a Fuzzy Steering Controller." IEEE Xplore. Institute of Electrical and Electronics Engineers, June 2011.

http:// ieeexplore.ieee.org/stamp/stamp.jsp?tp=\&arnu mber $=5715879$

[14] Wert, Ray. "Volvo Pedestrian Avoidance Test Fails Spectacularly." Jalopnik. N.p., 26 Sept. 2010.http://jalopnik.com/5648126/volvo-pedestrianavoidance-crash-test-fails-spectacularly 